\title{
Periodontal Diagnosis: Shall Saliva and Gingival Crevicular Fluid Help the Clinician?
}

\author{
Discepoli ${ }^{1 *}$, Mirra $\mathbf{R}^{1}$ and Marruganti $\mathbf{C}^{2}$ \\ ${ }^{1}$ Department of Medical Biotechnologies, University of Siena, Italy \\ ${ }^{2}$ Undergraduate Program in Dentistry, University of Siena, Italy
}

ISSN: 2637-7764

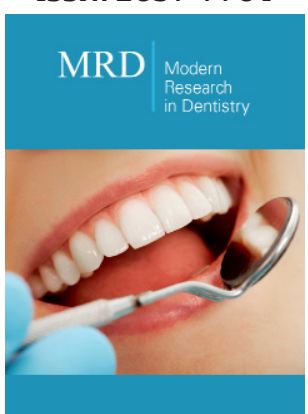

*Corresponding author: Discepoli Nicola, Department of Medical Biotechnologies, Unit of Periodontics, University of Siena, Siena, Italy

Submission: 此 March 16, 2020

Published: 佔 May 07, 2020

Volume 5 - Issue 1

How to cite this article: Discepoli N, Mirra R, Marruganti C. Periodontal Diagnosis: Shall Saliva and Gingival Crevicular Fluid Help the Clinician?. Mod Res Dent. 5(1) MRD.000604.2020.

DOI: 10.31031/MRD.2020.05.000604

Copyright@ Discepoli N, This article is distributed under the terms of the Creative Commons Attribution 4.0 International License, which permits unrestricted use and redistribution provided that the original author and source are credited.

\section{Introduction}

Periodontal diseases are a group of inflammatory/infectious, multifactorial, diseases. The periodontal tissues house both microbial dysbiosis and host response dysregulation [1]. The most recent hypothesis about the pathogenesis of periodontal disease deals with the biological transition from a healthy periodontal tissue to a pathological one (characterized by inflammation and loss of clinical attachment). This transition is mediated by the dysregulation of the inflammatory response, caused by the presence of keystone pathogens. These bacteria (i.e. Porphyromonas gingivalis) differ from normal commensal bacteria: they are able to alter the inflammatory response even in minimal quantities [2,3]. In fact, the proactivity of these species (keystone) increases the nososymbiocity of the dental biofilm without increasing its biomass [4].

This outbreak of the inflammatory response represents the pathological mechanism underlying periodontal disease. Individual variability in host response pathways may result in variations on the degree of inflammation, both in terms of response and resolution [5]. This feature, together with patient's behavioral habits, determine the heterogenic nuances (i.e. disease phenotypes) noticeable among individuals. Loss of clinical attachment (CAL loss) represents the pathognomonic sign of periodontitis: it yields two different clinical scenarios namely pocketing and gingival recession. Younger individuals seem to express loss of attachment through the latter mechanism, while pocketing becomes the main mode of disease progression as subjects get older [6]. Many longitudinal studies, dealing with the natural history of periodontal disease and carried out among different untreated populations, have highlighted a common pattern of disease progression [7-9]. Usually, it is relatively slow and site-specific: interproximal sites are more prone to be affected by pocketing, whilst midbuccal and mid-lingual sites mainly through recession [10]. Previous studies have reported that patients showing a high level of gingival inflammation and chronic bleeding on probing are more likely to develop destructive periodontal disease, while further relapses of the disease are best predicted by the current signs and symptoms [11]. The effectiveness of clinical diagnostic procedures for intercepting disease progression is minimal. The progression of Periodontitis, indeed, is not linear. Since the 80's, results from longitudinal studies on untreated subjects suggested the so called "Burst hypothesis", as a possible explanation to how clinical attachment loss takes place over time. This model describes the development of loss of attachment as an asynchronous alternance between sudden tissue loss ("burst") and phases of stability [12]. Recently, a new model was proposed to interpret disease progression $[13,14]$.

It is based on a Linear Mixed Model (LMM) analysis that is supposed to overcome some short comings (site and patient level source of errors, reliability) of the previously proposed model. Considering the clinical and methodological features of the existing procedures, the therapist is called to face a disease without the tools for pinpointing a true state of "activity" of the disease. In fact, the evaluation of clinical attachment loss (CAL loss) identifies sites that have already experienced disease. CAL loss, measured by probing pocket depth and recession, represents the history of the disease experienced by the patient, but it holds very low reliability regarding the current and future course of the disease. Due to its chronic nature, 
an early detection of disease and disease activity is of paramount importance. In this perspective, recent scientific evidence suggests how saliva and gingival crevicular fluid (GCF) could contribute to its early detection. These fluids are a copious source of biological biomarkers eventually able to identify, way before clinical diagnosis, an imbalance between the host response and the biofilm.

That being said, which biomarkers are suitable to help the clinician?

In this perspective, scientific community has paid close attention to both saliva and GCF. In 2018, a new classification system for periodontal diseases was introduced. The newly proposed framework entails the incorporation of future potential biomarkers in order to integrate the information provided by the standard clinical measures. A recent systematic review [15] analyzed 32 biomarkers through a meta-analytical approach to test their diagnostic ability: sensitivity and specificity were collected in otherwise healthy subjects. The most frequently studied salivary biomarkers were MMP-8, IL-1 beta, IL-6, MMP-9 and Hb. They all showed a good capability to detect periodontitis, highlighted by a sensitivity value of more than 70\%. Furthermore, IL1 b and MMP-9 displayed also a good specificity (around $80 \%$ ).

Among these bio products, MMP-8 deserves special interest. It is probably the most investigated marker. Moreover the market offers a chair side/point of care oral fluid test, based on the detection of MMP-8, that has shown promising results in identifying active periodontal tissue destruction among populations of different ethnicities and with comorbidities [16-18]. Recently, the saliva concentration of MMP-8 was also directly related to staging and grading [19]. The MMP-8 levels in mouth rinse were significantly lower among healthy patients compared to individuals with advanced periodontal destruction. The scenario regarding gingival crevicular fluid is quite similar to that described for saliva. Among the biomarkers with the highest level of evidence, MMP-8 displayed a good sensitivity and an excellent specificity $(76,7 \%$ and $92 \%$ respectively) according to recent metanalytic data [15].

\section{Conclusion}

From a clinical standpoint, the use of a biological marker as a diagnostic tool could play a pivotal role in the very first steps of diagnosis. The possibility of carrying out an initial "triage" to subsequently identify which cases deserve a supplementary diagnosis represents a very close and useful horizon for the clinician.

\section{References}

1. Meyle J, Chapple I (2015) Molecular aspects of the pathogenesis of periodontitis. Periodontology 2000 69(1): 7-17.

2. Hajishengallis G, Lamont RJ (2012) Beyond the red complex and into more complexity: The polymicrobial synergy and dysbiosis (PSD) model of periodontal disease etiology. Molecular Oral Microbiology 27(6): 409419.

3. Bostanci N, Bao K, Greenwood D, Silbereisen, Belibasakis GN (2019) Periodontal disease: From the lenses of light microscopy to the specs of proteomics and next-generation sequencing. $1^{\text {st }}$ edn, Elsevier Inc., Advances in Clinical Chemistry 93: 263-290.

4. Proctor D, Shelef KM, Gonzalez A, Davis CL, Dethlefsen L, et al. (2019) Microbial biogeography and ecology of the mouth and implications for periodontal diseases. Periodontology 2000 82(1): 26-41.

5. Van Dyke TE, Kornman KS (2008) Inflammation and factors that may regulate inflammatory response. J Periodontol 79(8 Suppl): 1503-1507.

6. Schätzle M, Löe H, Lang NP, Heitz Mayfield LJA, Bürgin W, et al. (2003) Clinical course of chronic periodontitis: III. Patterns, variations and risks of attachment loss. Journal of Clinical Periodontology 30(10): 909-918.

7. Loe H (1979) The natural history of periodontal disease in man. Journal of Periodontal Research 14(6): 526-540.

8. Goodson JM, Haffajee AD, Socransky SS (1984) The relationship between attachment level loss and alveolar bone loss. Journal of Clinical Periodontology 11(5): 348-359.

9. Baelum V, Luan WM, Chen X, Fejerskov O (1997) A 10-year study of the progression of destructive periodontal disease in adult and elderly Chinese. Journal of periodontology 68(11): 1033-1042.

10. Thomson (2006) Changes in periodontal disease experience from 26 to 32 years of age in a birth cohort. Journal of Periodontology 23(1): 1-7.

11. Haffajee AD, Socransky SS, Lindhe J, Kent RL, Okamoto H et al. (1991) Clinical risk indicators for periodontal attachment loss. Journal of Clinical Periodontology 18(2): 117-125.

12. Goodson JM, Tanner AC, Haffajee AD, Sornberger GC, Socransky SS, et al. (1982) Patterns of progression and regression of advanced destructive periodontal disease. Journal of Clinical Periodontology 9(6): 472-481.

13. Teles R, Benecha HK, Preisser JS, Moss K, Starr JR, et al. (2016) Modelling changes in clinical attachment loss to classify periodontal disease progression. Journal of Clinical Periodontology 43(5): 426-434.

14. Teles R, Moss K, Preisser JS, Genco R, Giannobile WV, et al. (2018) Patterns of periodontal disease progression based on linear mixed models of clinical attachment loss. Journal of Clinical Periodontology 45(1): $15-25$

15. Arias Bujanda N, Regueira Iglesias A, Balsa Castro C, Nibali L, Donos N, et al. (2019) Accuracy of single molecular biomarkers in gingival crevicular fluid for the diagnosis of periodontitis: A systematic review and metaanalysis. Journal of Clinical Periodontology 46(12): 1166-1182.

16. Nwhator SO, Ayanbadejo PO, Umeizudike KA, Opeodu OI, Agbelusi GA, et al. (2014) Clinical correlates of a lateral-flow immunoassay oral risk indicator. Journal of Periodontology 85(1): 188-194.

17. Johnson N, Ebersole JL, Kryscio RJ, Danaher RJ, Dawson D, et al. (2016) Rapid assessment of salivary MMP-8 and periodontal disease using lateral flow immunoassay. Oral Diseases 22(7): 681-687.

18. Grigoriadis A, Sorsa T, Räisänen I, Pärnänen P, Tervahartiala T, et al. (2019) Prediabetes/diabetes can be screened at the dental office by a low-cost and fast chair-side/point-of-care aMMP-8 immunotest. Diagnostics 9(4).

19. Sorsa T, Alassiri S, Grigoriadis A, Räisänen IT, Pärnänen P, et al. (2020) Active MMP-8 (aMMP-8) as a grading and staging biomarker in the periodontitis classification. Diagnostics 10(2): 61. 